\title{
Unicompartmental Knee Arthroplasty: Modes of Failure and Conversion to Total Knee Arthroplasty
}

\author{
Michele Vasso ${ }^{1}$ Katia Corona ${ }^{1}$ Rocco D’Apolito ${ }^{1}$ Giuseppe Mazzitelli ${ }^{1}$ Alfredo Schiavone Panni ${ }^{2}$ \\ ${ }^{1}$ Department of Medicine and Health Sciences, University of Molise, \\ Campobasso, Italy \\ 2 Multidisciplinary Department of Medical, Surgical and Dental \\ Sciences, University Campana Luigi Vanvitelli, Caserta, Italy \\ Address for correspondence Michele Vasso, MD, Department of \\ Medicine and Health Sciences, University of Molise, Via Francesco De \\ Sanctis, 86100, Campobasso, Italy (e-mail: vassomichele@gmail.com). \\ Joints 2017;5:44-50

\begin{abstract}
Despite the excellent success rates of the modern unicompartmental knee arthroplasty (UKA), results of knee replacement registries still shows a relatively high revision and

Keywords

- unicompartmental knee arthroplasty

- polyethylene

- loosening

- bearing dislocation

- revision

- conversion failure rate for UKA, especially when compared with traditional total knee arthroplasty (TKA). Bearing dislocation continues to be advocated as the predominant mechanism of failure in mobile UKA, whereas polyethylene wear and aseptic loosening remains the main cause of failure of fixed UKA. Degeneration of the unreplaced compartments has been reported in both mobile and fixed designs. When the revision is required, most of failed UKAs are converted to TKAs. Surgical challenges of the UKA revision, and outcomes of UKA converted to TKA are still debated in literature.
\end{abstract}

\section{Introduction}

During the last 20 years, the use of unicompartmental knee arthroplasty (UKA) has considerably increased. Between 1998 and 2005, use of UKA increased at a rate nearly triple that of total knee arthroplasty (TKA). ${ }^{1}$ UKA can be performed through a minimally invasive approach and is associated with a relatively low incidence of complications. ${ }^{2,3}$ Several reports have already demonstrated survival rates greater than $90 \%$ at 10 years after modern UKA implantation. ${ }^{1,4-7}$ Despite this, results of knee replacement registries still show a relatively high revision and failure rate for UKA, especially when compared with traditional TKA. ${ }^{8}$ Failures of UKAs have distinctive characteristics, and consensus on the causes and the proper treatment methods has been elusive. Additionally, the incidence and type of complications are different, depending on the type and design of implant, follow-up period, and the surgeon who performed the procedure. ${ }^{9}$ When the revision is required, most UKAs are converted to TKAs; surgical challenges of UKA revisions, and outcomes of UKA conversion to TKA appear still controversial.

The purpose of this concept review was to analyze the recent literature to elucidate the main causes and mechanisms of UKA failure, the principal surgical aspects that should be considered during UKA revision, and the main outcomes of UKA conversion to TKA.

\section{Causes of Unicompartmental Knee Arthroplasty Failure}

The main causes of UKA failure include bearing dislocation (in mobile designs), aseptic mechanical loosening, polyethylene wear, progression of osteoarthritis (OA) in unreplaced compartments, infection, impingement, periprosthetic fracture, retaining of cement debris into the joint, arthrofibrosis, and unexplained pain. ${ }^{1,9-11}$ Bearing dislocation continues to be advocated as the predominant mechanism of failure in mobile UKA, 2,9,12 whereas polyethylene wear and aseptic loosening remains the main cause of failure of fixed UKA. ${ }^{1,9}$ Degeneration of the unreplaced compartments has been reported in both mobile and fixed designs. Regardless of the implant design, most of the failures occur in medial UKA when compared with lateral UKA. ${ }^{9}$

\section{Bearing Dislocation}

Bearing dislocation is certainly the major complication of mobile UKA. Possible causes of insert dislocation could be malposition of the components, unbalanced flexion-
DOI http://dx.doi.org/ 10.1055/s-0037-1601414. ISSN 2282-4324.
Copyright @ 2017 Georg Thieme Verlag KG Stuttgart · New York
License terms

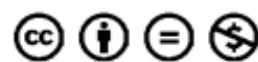


extension gap, impingement of the insert with adjacent bone or tibial/femoral component, or instability due to medial collateral ligament (MCL) injury or secondary to femoral/ tibial component loosening. 9,13 Dislocations are particularly common in lateral UKA, as the lateral collateral ligament is slack in flexion, in contrast to the medial side in which the MCL is tight. ${ }^{12,14}$

MCL is the most important structure determining the flexion-extension gap and affecting stability (with the anterior cruciate ligament) after UKA. Therefore, the surgeon should take utmost care to protect the MCL during surgery. For the same reasons, in UKA, soft tissue release should never be performed. The aim of UKA is to restore knee kinematics by restoring ligament tension to normal; correct ligament balance is restored by positioning the components accurately and inserting an appropriate thickness of bearing. ${ }^{3}$ On the contrary, ligament release may result in excessive laxity of the periprosthetic soft tissues, with the consequent trend of the insert (and implant) to dislocate anteriorly. Additionally, this may cause early polyethylene wear as well as aseptic loosening. ${ }^{15}$

Bearing dislocation can be treated with bearing change, revision UKA, or conversion to TKA, according to the cause of dislocation.

\section{Aseptic Mechanical Loosening}

While aseptic mechanical loosening has been recently reported as the most frequent cause of failure of the modern TKA, ${ }^{16}$ newer instrumentation and prosthetic design have significantly reduced its incidence in modern UKA. Younger age, overweight, and varus deformity have been advocated as possible risk factors for mechanical failure of unicompartmental implants. Fixed-bearing UKA results in greater contact stress on the polyethylene insert due to low conformity, which may eventually lead to a failure associated with tibial component loosening or subsidence. ${ }^{9}$ Moreover, it is known that tibial component loosening is more frequent in the allpoly designs than the metal-backed ones. ${ }^{17}$ Mechanical loosening following UKA may be potentially determined by component malalignment, undercorrection of the predegeneration deformity, anterior cruciate ligament deficiency, excessive tibial slope, and bearing dislocation in mobile designs. Additionally, all these factors may also produce wear-induced periprosthetic osteolysis (due to an increased asymmetrical loading), with a further increase of the component subsidence and/or loosening. ${ }^{15}$

Component loosening should be treated with a revision UKA or a conversion to TKA, according to the cause of loosening and the residual integrity of bone stock and ligaments.

\section{Progression of Osteoarthritis}

Progression of $\mathrm{OA}$ in the contralateral compartment and/or in the patellofemoral joint (PFJ) is one of the major causes of failure following UKA, both mobile and fixed. Overcorrection of the leg mechanical axis may cause degenerative changes in the contralateral compartment. ${ }^{3}$ Progression of OA may occur in case of patients with systemic inflammatory dis- eases, that is, rheumatoid or psoriatic arthritis. Degeneration of PFJ may occur in the presence of an oversized femoral component with possible impingement with patellar cartilage. ${ }^{1}$ Progression of $\mathrm{OA}$ in the contralateral and/or patellofemoral compartment can be characterized by radiographic evidence of joint space narrowing and osteophyte formation in the initial stage, which eventually leads to development of pain, subchondral sclerosis, and loss of joint space in unreplaced compartments. ${ }^{18}$

Progression of OA should be treated with replacement of the newly affected compartment (bi- or tri-UKA) or with conversion to TKA.

\section{Polyethylene Wear}

Polyethylene wear is a complication inherent to the design of fixed bearings, secondary to higher surface deformation and delamination in comparison to mobile bearings. ${ }^{19}$ Revision for polyethylene wear usually occur after a minimum of 8 years, but some early catastrophic failure from wear have been reported. ${ }^{15}$ Polyethylene wear increases the debris volume at the bone-implant interface, producing osteolysis, which plays a role in component loosening and degeneration of unreplaced compartments. Additionally, wear affects joint alignment and stability, leading to an increased load at the bone-implant interface that further accelerates loosening. ${ }^{15}$

Factors associated with accelerated polyethylene wear after UKA are component malpositioning, undercorrection of the predegeneration deformity, polyethylene thickness of less than $6 \mathrm{~mm}$, manufacturing process and sterilization method of polyethylene, and reduced conformity in the design. ${ }^{12,15}$ Modern instrumentation helps to avoid component malposition and edge loading, even with a minimally invasive approach. Furthermore, polyethylene is now thicker than $6 \mathrm{~mm}$ for fixed bearings, and the shelf age of polyethylene inserts sterilized with gamma irradiation in air is well-documented. Finally, recent improvements in manufacturing processes such as cross-linking may be valuable in some fixed and mobile designs.

If a failure occurs due to polyethylene wear, insert change can be considered as treatment choice; however, conversion to TKA is the most common option in these cases.

\section{Periprosthetic Infection}

Incidence of infection after UKA is significantly lower than that after TKA, varying from 0.2 to $1 \%{ }^{20,21}$ The low incidence of infection in UKA is because it can be performed through a minimally invasive approach, with less damage to the adjacent soft tissue and sparing of bone and ligamentous structures.

During periprosthetic infection, cellular mechanisms of osteolysis and septic loosening can be determined by the direct damage of infectious organisms and by the host inflammatory response, mainly due to bacterial toxins and lipopolysaccharides. $^{22}$

In patients with an acute UKA infection, the strategy of irrigation and debridement with insert exchange still persists, given the emotional investment in dealing with this complication by both patient and surgeon. In failed 
debridement or in chronic infections, resection of all components is necessary, and a one-stage or two-stage revision surgery with a primary TKA should be performed.

\section{Periprosthetic Fracture}

Periprosthetic fractures are rare but represent a serious complication in UKA. They are mostly observed around the tibial condyles; this can be attributed to the increased pressure and load applied on the proximal tibia. A tibial condylar fracture following UKA can be managed with conservative treatment if slight translation or varus deformity is present. Rarely, periprosthetic fracture of the femoral condyle may occur; factors leading to this complication could be the impaction force or direction, or a diminished load resistance of the distal femur. ${ }^{23}$ If the fracture is accompanied by translation or deformity, percutaneous osteosynthesis with cannulated screws, or open reduction and internal fixation with plates should be performed. ${ }^{24}$ In case of nonunion of the fracture, severe translation, or tibial component loosening, UKA needs to be revised to TKA. ${ }^{9}$

\section{Arthrofibrosis}

The incidence of arthrofibrosis after UKA is much lower than that after TKA because minimally invasive procedure causes reduced damage to the extensor mechanism and suprapatellar pouch and lesser scar formation. ${ }^{25}$ Additionally, modern designs of UKA have greatly reduced the incidence of prosthetic impingement and/or impingement of the femoral component with the patella.

Postoperative arthrofibrosis in UKA can be treated with manipulation under anesthesia, which often requires arthroscopic removal of intra-articular fibrous tissue and scar tissue.

\section{Unexplained Pain}

Revision for unexplained pain has been reported more common after UKA than after TKA. ${ }^{26}$ Common causes may include loose bodies, chronic regional pain syndrome, implant failure, meniscus rupture, and cement extrusion. Additionally, unexplained pain may be caused by subtle problems that the surgeon is unable to document. Revision for unexplained pain after UKA may be related to the surgeon's experience. ${ }^{13}$ Finally, failure due to unexplained pain has been particularly reported in UKAs with all-polyethylene tibial components, which may increase load transfer to the proximal tibia, thus resulting in persistent bone remodeling. ${ }^{19}$

\section{Unicompartmental Knee Arthroplasty Revision: Surgical Aspects}

Depending on the type, severity, and consequences of UKA failure, revision surgery might be indicated, such as simple polyethylene insert exchange, revision UKA, or conversion to TKA.

Polyethylene insert exchange could be indicated in case of insert dislocation or wear associated with stable components and no severe osteolysis. Revision UKA can be performed in the presence of complete ligament integrity and sufficient bone stock; obviously, there must be no signs of degeneration of unreplaced compartments. Nevertheless, most of the failed UKAs are generally converted to TKA. ${ }^{13,27,28}$ The reasons for conversions to TKA are repeated dislocation in a mobile UKA, mechanical loosening with associated bone defect, visible torn anterior cruciate ligament, valgus overcorrection, and progression of $\mathrm{OA}$ in the lateral and/or patellofemoral compartment. $^{13}$

Conversion to TKA may be technically demanding, especially in septic revisions. ${ }^{20}$ Preoperative planning is mandatory to anticipate the need for revision components as well as the availability of constrained implants. ${ }^{29}$ Challenges of UKA revision can be joint exposure, component removal, bone defects, and instability.

Regarding exposure, there is generally no necessity of quadriceps snip, V-Y plasty, or tibial tuberosity osteotomy, and a standard approach can be considered according to surgeon's preference.

Although unicompartmental components can be easily removed with an osteotome or a thin blade saw, the surgeon should be careful to preserve bone stock. In the presence of an uncemented prosthesis, removal of a stable implant could cause significant bone loss, whereas removal of a loosed implant generally preserves much more bone stock. In the presence of a cemented prosthesis, implant removal is usually easier because a detachment usually occurs at the cement-metal interface.

Bone defects may occur at the site of a failed UKA, so conversion to TKA may require revision devices such as metal augments, bone grafts, and stems. Causes of bone loss after the UKA failure could be metal-backed design (when compared with all-polyethylene tibia), osteolysis from wear, periprosthetic infection, loosening with component subsidence, removal of components and/or cement, periprosthetic fracture, and the presence and successive removal of spacer in septic two-stage conversion. In most cases of UKA failure, residual femoral defects are generally small and contained, thus requiring only autologous morcellized bone grafts or cement. At revision, correct femoral component rotation can be established before removing the UKA; otherwise, the epicondylar and transtrochlear axes can be also established after removing the UKA. ${ }^{30}$ More frequently, in a failed UKA, bone loss affects the tibial side, primarily due to the native tibial bone cut (especially in metal-backed implants). On the preoperative radiographs, if the line orthogonal to the mechanical axis drawn $10 \mathrm{~mm}$ below the joint line of the unaffected compartment lies below the tibial component, then an augmentation will not be necessary (-Fig. 1). On the contrary, if the defect is greater than $10 \mathrm{~mm}$, then the surgeon could necessitate a metal block/wedge or a bone graft; in this case, a tibial stem may be recommended (- Fig. 2). ${ }^{11}$ Finally, mediolateral stability in the coronal plane is generally preserved in failed UKA, thus allowing the surgeon to use a primary TKA, with or without posterior ligament sacrifice. Anyway, when there is deficiency of collateral ligaments, soft tissue instability, and inability to balance the flexion-extension spaces, a more constrained prosthesis is necessary. 

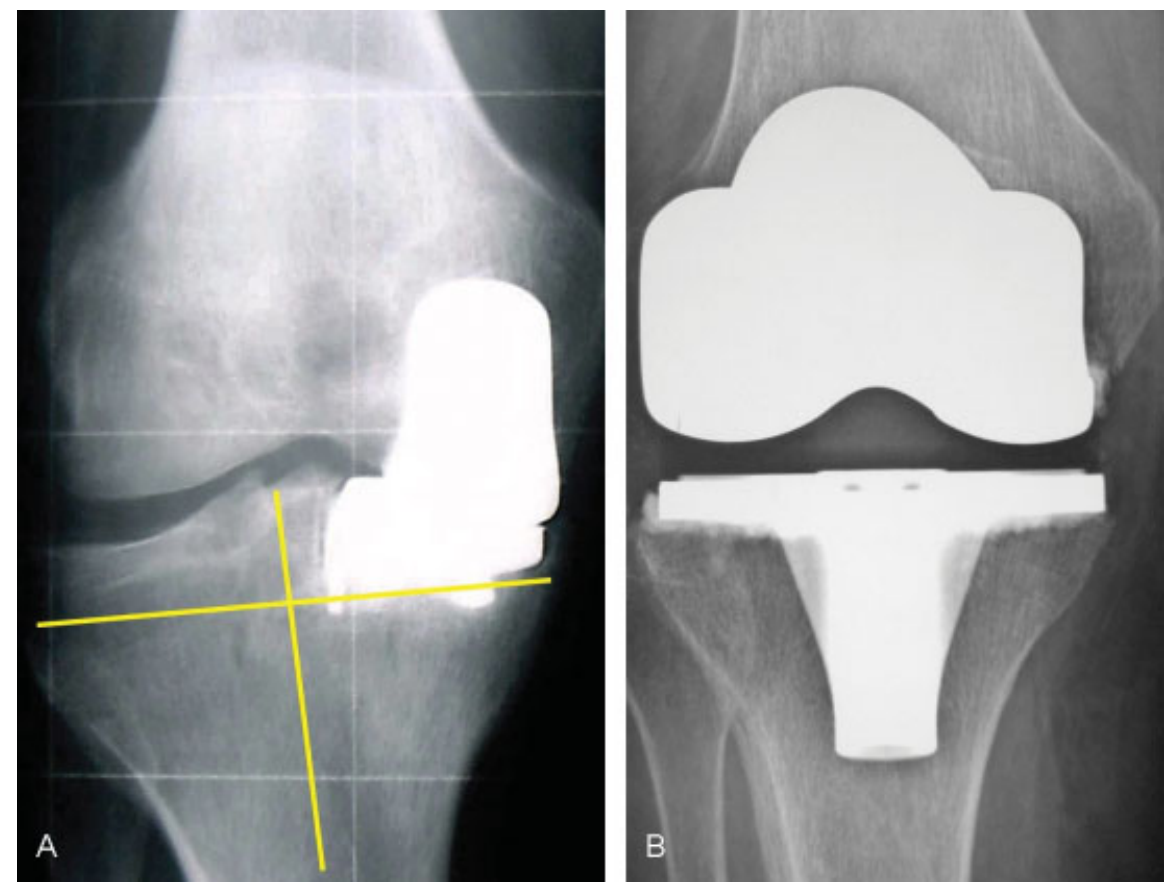

Fig. 1 When the line orthogonal to the mechanical axis drawn $10 \mathrm{~mm}$ below the joint line of the unaffected compartment lies below the tibial component $(A)$, then tibial augmentation is not generally necessary and a primary tibial component is sufficient (B).

\section{Literature Review}

Complications following UKA are various and depend on the design and type of implant, follow-up period, and the surgeon who performed the surgery. ${ }^{9}$
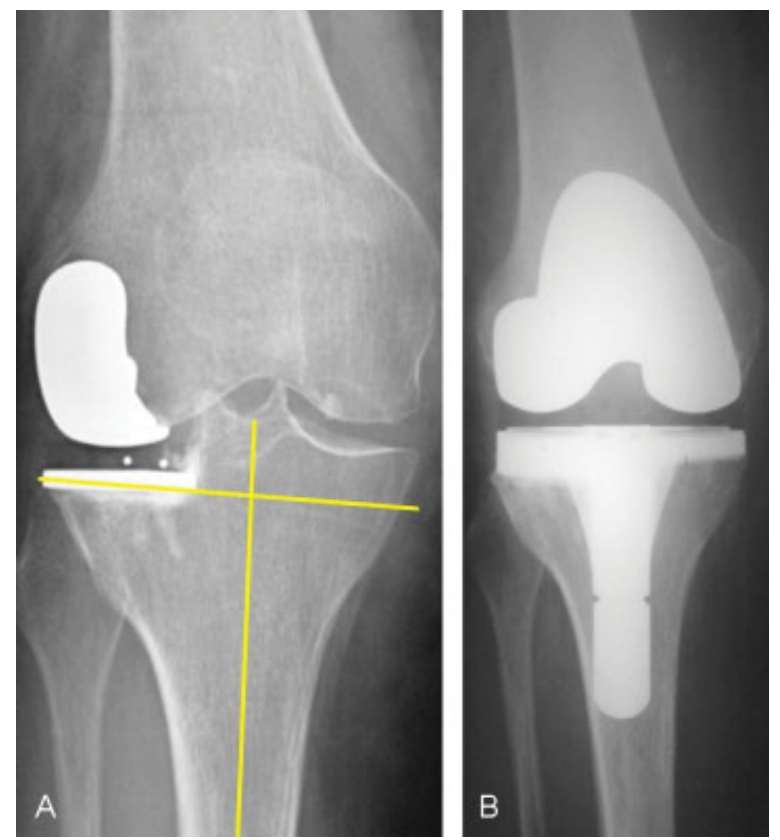

Fig. 2 When the line orthogonal to the mechanical axis drawn $10 \mathrm{~mm}$ below the joint line of the unaffected compartment lies inside the tibial component $(A)$, then a metal block or wedge can be used during revision to avoid an excessive tibial resection and therefore the use of an excessively thick polyethylene insert. In this case, it is also recommended to add a short stem extension to decrease stress at the bone-implant interface (B).
Pandit et $\mathrm{al}^{31}$ reported a $2.9 \%$ rate of complications after 1,000 Oxford phase 3 medial UKAs at a mean follow-up of 5.6 years. In their study, the most common causes for revision were progression of $\mathrm{OA}$ in the lateral compartment $(0.9 \%)$, bearing dislocation $(0.6 \%)$, and unexplained pain $(0.6 \%)$. Of the 29 reoperations, 19 were conversions to a primary TKA ( 2 of those required revision devices as stems and wedges), 6 were open reductions for dislocation of the bearing, 3 were secondary lateral UKA, and 1 was revision of a tibial component.

Epinette et $\mathrm{al}^{32}$ in a multicenter study including 418 failed UKAs reported loosening as the main reason for failure (45\%), followed by OA progression (15\%) and wear (12\%). Other reasons were technical problems in $11.5 \%$ of cases, unexplained pain in 5.5\%, and failure of the supporting bone in $3.6 \%$. The infection rate was $1.9 \%$. Recently, Kim et $\mathrm{al}^{9}$ reported a total of 89 (5.6\%) failures on 1,576 UKAs. The causes of failure included bearing dislocation $(n=42)$, component loosening $(n=23)$, femoral component loosening $(n=11)$, tibial component loosening $(n=8)$, both femoral and tibial component loosening $(n=4)$, periprosthetic fracture $(n=6)$, polyethylene wear/destruction $(n=3)$, progression of arthritis to the other compartment $(n=3)$, MCL injury $(n=2)$, impingement $(n=2)$, infection $(n=5)$, ankylosis $(n=1)$, and unexplained pain $(n=2)$. The most common complication in mobile UKA was bearing dislocation, and in fixed UKA, it was mechanical loosening. Polyethylene wear and progression of OA were relatively rare. Complications were treated with conversion to TKA in 58 cases, bearing change in 21 , and revision with another UKA in 1. In all the cases of conversion to TKA (including infected UKAs), a primary TKA was used; in $74 \%$ of the conversions to TKA, a metal augmentation was used. 
In a systematic review of 17 published studies that evaluated the causes of reoperation after UKA using the Oxford phase 3 implant, Kim et $\mathrm{al}^{13}$ assessed a total of 3,138 UKAs with a median follow-up period was 5.6 years. UKA failure occurred in 146 (4.6\%) knees. Bearing dislocation was found to be the most important cause of revision, with a pooled percentage of $1.5 \%$ (47/3,138 knees). The rate of bearing dislocation was significantly higher in Asian population than that in western population. Loosening of either the tibial or femoral component was the second most common reason for further surgical intervention (1\%). Persistent pain ( $0.8 \%)$, OA of the lateral compartment $(0.5 \%)$, infection $(0.3 \%)$, and medial tibial plateau fracture $(0.2 \%)$ were the remaining causes of failure.

In a systematic review and meta-analysis, Peersman et $\mathrm{al}^{28}$ reported the outcomes of 4,330 fixed-bearing and 5,133 mobile-bearing UKAs. Mean follow-up was 8.7 years for fixed UKAs and 5.9 years for mobile UKAs. Although mobile and fixed designs presented comparable revision rates, major complications among fixed implants were progression of OA (0.29\%) and component loosening (0.22\%), whereas those among mobile implants were component loosening $(0.44 \%)$, bearing dislocation $(0.29 \%)$, and progression of arthritis (0.23\%).

In a systematic review, Ko et $\mathrm{al}^{27}$ found that the overall reoperation rate and the overall incidence of complications were similar between the mobile bearings $(1,392)$ and fixed bearings (1,377); however, mobile bearings were more susceptible to reoperations due to aseptic loosening, progression of OA, and implant dislocation. Major incidence of component loosening reported in mobile UKA was associated with osteolysis due to wear of submicron particles typical of mobile-bearing designs; these smaller wear-particles are biologically more active and have high propensity for osteolysis.

Conversion of a failed UKA to TKA could be a demanding procedure. Technical difficulty may depend on how conservative the initial procedure was and on the primitive mode of failure and its consequences on bone stock and ligament integrity, often with the need for metal augmentation and stems. $^{29,30}$

Controversies still exist about functional outcomes and final survivorship of TKA after a failed UKA. Studies on conversion to TKA of failed first-generation UKAs reported poorer results than those of primary TKA and comparable to revision TKA. ${ }^{33-35}$ On the contrary, conversion of secondgeneration UKA resulted in better outcomes and therefore comparable to primary TKA. ${ }^{11,36}$

Some authors demonstrated how the cause of UKA failure could affect outcomes of the successive conversion to TKA. Kerens et $\mathrm{al}^{37}$ found that conversion of UKA to TKA for unexplained pain generally resulted in a less favorable outcome than revision for a known cause of pain. Furthermore, studies ${ }^{11,38}$ where the reason of revision was mostly progression of $\mathrm{OA}$, polyethylene wear without loosening, or polyethylene dislocation showed better outcomes than studies where loosening was the primary reason of conversion. ${ }^{39,40}$
Lunebourg et $\mathrm{al}^{33}$ reported that the main reason of revision was progression of $\mathrm{OA}$ and that the results of revised UKA with TKA were close to those of revised TKA. The authors compared 48 UKAs converted to TKA, 48 primary TKAs, and 48 revision TKAs. The groups were matched for age, gender, body mass index, preoperative Knee Society Score, length of follow-up, and date of the index surgery. Surgical characteristics, clinical outcomes, and complications were compared at a mean follow-up of 7 years. Regarding the 48 converted UKAs, 43 patients received a primary posterior-stabilized TKA, whereas 5 patients received a semiconstrained implant. An autologous graft was used in 6 patients, a tibial metal augment in 14 patients, and a tibial stem in 35 patients. The authors concluded that even if the conversion of UKA was technically less demanding than a revision TKA, functional scores, quality of life, complications, and survival rate after revision UKA were more comparable to revision TKA than to primary TKA.

Järvenpää et $\mathrm{al}^{41}$ compared the long-term clinical and functional results of 21 UKAs converted to TKA with those of 28 primary TKAs matched for age, sex, and operation time point. The mean follow-up period of the patients was 10.5 years. The UKA-converted patients were less satisfied, as measured by the WOMAC (Western Ontario and McMaster Universities Arthritis Index) scale. Two patients were revised twice in the UKA conversion group. There was one revision in the primary TKA group. Improvement in range of motion was better in the TKA patients compared with the UKA conversion patients. The authors concluded that UKA conversion to TKA is associated with poorer clinical outcome as compared with primary TKA.

Similarly, Craik et $\mathrm{al}^{42}$ in 25 UKAs revised with TKA, found that 10 (34\%) patients required augments, stemmed implants, or bone grafts. Outcomes following UKA converted to TKA were poorer than those following successful UKA and primary TKA, albeit these results were found to be a consequence of poor preoperative function of the failed-UKA patients.

Jonas et $\mathrm{al}^{43}$ concluded that revision of UKA with TKA is not a straightforward procedure comparable to a standard primary knee replacement. Additionally, despite the cost of components not being significantly higher than that in primary TKA and despite the clinical outcomes being similar at 5 years, there were multiple hidden follow-up costs in converted UKAs.

Finally, Cankaya and Della Valle ${ }^{44}$ found that conversion of a failed UKA is similar to primary TKA with respect to blood loss and transfusion rate.

\section{Conclusion}

Although incidence of failure is greatly diminished in the last generation UKA, bearing dislocation, polyethylene wear, mechanical loosening, and progression of OA continue to affect the final survivorship of contemporary UKA. Converting a UKA to TKA is more complicated than performing a primary TKA, and clinical and functional results after revision TKA for a failed UKA could be inferior to those of a 
primary TKA. A primary implant can be used to convert a failed UKA to a TKA; however, surgeons should be aware that in revision UKAs, stems and augments on the tibial side are often required.

\section{References}

1 Foran JR, Brown NM, Della Valle CJ, Berger RA, Galante JO. Longterm survivorship and failure modes of unicompartmental knee arthroplasty. Clin Orthop Relat Res 2013;471(01):102-108

2 Bergeson AG, Berend KR, Lombardi AV Jr, Hurst JM, Morris MJ, Sneller MA. Medial mobile bearing unicompartmental knee arthroplasty: early survivorship and analysis of failures in 1000 consecutive cases. J Arthroplasty 2013;28(9, Suppl):172-175

3 Vasso M, Del Regno C, D’Amelio A, Viggiano D, Corona K, Schiavone Panni A. Minor varus alignment provides better results than neutral alignment in medial UKA. Knee 2015;22(02):117-121

4 Koshino T, Sato K, Umemoto Y, Akamatsu Y, Kumagai K, Saito T. Clinical results of unicompartmental arthroplasty for knee osteoarthritis using a tibial component with screw fixation. Int Orthop 2015;39(06):1085-1091

5 Schlueter-Brust K, Kugland K, Stein G, et al. Ten year survivorship after cemented and uncemented medial Uniglide ${ }^{\circledR}$ unicompartmental knee arthroplasties. Knee 2014;21(05):964-970

6 Vasso M, Del Regno C, Perisano C, D’Amelio A, Corona K, Schiavone Panni A. Unicompartmental knee arthroplasty is effective: ten year results. Int Orthop 2015;39(12):2341-2346

7 Yoshida K, Tada M, Yoshida H, Takei S, Fukuoka S, Nakamura H. Oxford phase 3 unicompartmental knee arthroplasty in Japanclinical results in greater than one thousand cases over ten years. J Arthroplasty 2013;28(9, Suppl):168-171

8 Niinimäki TT, Murray DW, Partanen J, Pajala A, Leppilahti JI. Unicompartmental knee arthroplasties implanted for osteoarthritis with partial loss of joint space have high re-operation rates. Knee 2011;18(06):432-435

$9 \mathrm{Kim}$ KT, Lee S, Lee JI, Kim JW. Analysis and treatment of complications after unicompartmental knee arthroplasty. Knee Surg Relat Res 2016;28(01):46-54

10 Clark M, Campbell DG, Kiss G, Dobson PJ, Lewis PL. Reintervention after mobile-bearing Oxford unicompartmental knee arthroplasty. Clin Orthop Relat Res 2010;468(02):576-580

11 Springer BD, Scott RD, Thornhill TS. Conversion of failed unicompartmental knee arthroplasty to TKA. Clin Orthop Relat Res 2006;446(446):214-220

12 Weston-Simons JS, Pandit H, Gill HS, et al. The management of mobile bearing dislocation in the Oxford lateral unicompartmental knee replacement. Knee Surg Sports Traumatol Arthrosc 2011; 19(12):2023-2026

13 Kim SJ, Postigo R, Koo S, Kim JH. Causes of revision following Oxford phase 3 unicompartmental knee arthroplasty. Knee Surg Sports Traumatol Arthrosc 2014;22(08):1895-1901

14 Price AJ, Svard U. A second decade lifetable survival analysis of the Oxford unicompartmental knee arthroplasty. Clin Orthop Relat Res 2011;469(01):174-179

15 Argenson JN, Parratte S. The unicompartmental knee: design and technical considerations in minimizing wear. Clin Orthop Relat Res 2006;452(452):137-142

16 Dalury DF, Pomeroy DL, Gorab RS, Adams MJ. Why are total knee arthroplasties being revised? J Arthroplasty 2013;28(8, Suppl): 120-121

17 Collier MB, Eickmann TH, Sukezaki F, McAuley JP, Engh GA. Patient, implant, and alignment factors associated with revision of medial compartment unicondylar arthroplasty. J Arthroplasty 2006;21(06, Suppl 2):108-115

18 Pandit H, Hamilton TW, Jenkins C, Mellon SJ, Dodd CA, Murray DW. The clinical outcome of minimally invasive Phase 3 Oxford unicompartmental knee arthroplasty: a 15-year follow-up of 1000 UKAs. Bone Joint J 2015;97-B(11):1493-1500

19 Bhattacharya R, Scott CE, Morris HE, Wade F, Nutton RW. Survivorship and patient satisfaction of a fixed bearing unicompartmental knee arthroplasty incorporating an all-polyethylene tibial component. Knee 2012;19(04):348-351

20 Labruyère C, Zeller V, Lhotellier L, et al. Chronic infection of unicompartmental knee arthroplasty: one-stage conversion to total knee arthroplasty. Orthop Traumatol Surg Res 2015; 101(05):553-557

21 Vasso M, Schiavone Panni A, De Martino I, Gasparini G. Prosthetic knee infection by resistant bacteria: the worst-case scenario. Knee Surg Sports Traumatol Arthrosc 2016;24(10):3140-3146

22 Vasso M, Schiavone Panni A. Low-grade periprosthetic knee infection: diagnosis and management. J Orthop Traumatol 2015;16(01):1-7

23 Ten Brinke B, de Haan LJ, Koenraadt KL, van Geenen RC. Medial femoral condyle fracture as an intraoperative complication of Oxford unicompartmental knee replacement. Knee Surg Sports Traumatol Arthrosc 2016;24(10):3191-3193

24 Seeger JB, Jaeger S, Röhner E, Dierkes H, Wassilew G, Clarius M. Treatment of periprosthetic tibial plateau fractures in unicompartmental knee arthroplasty: plates versus cannulated screws. Arch Orthop Trauma Surg 2013;133(02):253-257

25 Pandit H, Jenkins C, Barker K, Dodd CA, Murray DW. The Oxford medial unicompartmental knee replacement using a minimallyinvasive approach. J Bone Joint Surg Br 2006;88(01):54-60

26 Baker PN, Petheram T, Avery PJ, Gregg PJ, Deehan DJ. Revision for unexplained pain following unicompartmental and total knee replacement. J Bone Joint Surg Am 2012;94(17):e126. Doi: 10.2106/JBJS.K.00791

27 Ko YB, Gujarathi MR, Oh KJ. Outcome of unicompartmental knee arthroplasty: a systematic review of comparative studies between fixed and mobile bearings focusing on complications. Knee Surg Relat Res 2015;27(03):141-148

28 Peersman G, Stuyts B, Vandenlangenbergh T, Cartier P, Fennema P. Fixed-versus mobile-bearing UKA: a systematic review and metaanalysis. Knee Surg Sports Traumatol Arthrosc 2015;23(11): 3296-3305

29 Berend KR, George J, Lombardi AV Jr. Unicompartmental knee arthroplasty to total knee arthroplasty conversion: assuring a primary outcome. Orthopedics 2009;32(09). Doi: 10.3928/ 01477447-20090728-32

30 Khan Z, Nawaz SZ, Kahane S, Esler C, Chatterji U. Conversion of unicompartmental knee arthroplasty to total knee arthroplasty: the challenges and need for augments. Acta Orthop Belg 2013; 79(06):699-705

31 Pandit H, Jenkins C, Gill HS, Barker K, Dodd CA, Murray DW. Minimally invasive Oxford phase 3 unicompartmental knee replacement: results of 1000 cases. J Bone Joint Surg Br 2011; 93(02):198-204

32 Epinette JA, Brunschweiler B, Mertl P, Mole D, Cazenave A; French Society for Hip and Knee. Unicompartmental knee arthroplasty modes of failure: wear is not the main reason for failure: a multicentre study of 418 failed knees. Orthop Traumatol Surg Res 2012;98(6, Suppl)S124-S130

33 Lunebourg A, Parratte S, Ollivier M, Abdel MP, Argenson JN. Are revisions of unicompartmental knee arthroplasties more like a primary or revision TKA? J Arthroplasty 2015;30(11): 1985-1989

34 Barrett WP, Scott RD. Revision of failed unicondylar unicompartmental knee arthroplasty. J Bone Joint Surg Am 1987;69(09): 1328-1335

35 Lai CH, Rand JA. Revision of failed unicompartmental total knee arthroplasty. Clin Orthop Relat Res 1993;(287):193-201

36 Leta TH, Lygre SH, Skredderstuen A, et al. Outcomes of unicompartmental knee arthroplasty after aseptic revision to total knee arthroplasty: a comparative study of 768 TKAs and 578 UKAs 
revised to TKAs from the Norwegian arthroplasty register (1994 to 2011). J Bone Joint Surg Am 2016;98(06):431-440

37 Kerens B, Boonen B, Schotanus MGM, Lacroix H, Emans PJ, Kort NP. Revision from unicompartmental to total knee replacement: the clinical outcome depends on reason for revision. Bone Joint J 2013;95-B(09):1204-1208

38 Johnson S, Jones P, Newman JH. The survivorship and results of total knee replacements converted from unicompartmental knee replacements. Knee 2007;14(02):154-157

39 Châtain F, Richard A, Deschamps G, Chambat P, Neyret P. Revision total knee arthroplasty after unicompartmental femorotibial prosthesis: 54 cases [in French]. Rev Chir Orthop Repar Appar Mot 2004;90(01):49-57

40 Sierra RJ, Kassel CA, Wetters NG, Berend KR, Della Valle CJ, Lombardi AV. Revision of unicompartmental arthroplasty to total knee arthroplasty: not always a slam dunk!. J Arthroplasty 2013; 28(8, Suppl):128-132
41 Järvenpää J, Kettunen J, Miettinen H, Kröger H. The clinical outcome of revision knee replacement after unicompartmental knee arthroplasty versus primary total knee arthroplasty: 8-17 years follow-up study of 49 patients. Int Orthop 2010;34(05):649653

42 Craik JD, El Shafie SA, Singh VK, Twyman RS. Revision of unicompartmental knee arthroplasty versus primary total knee arthroplasty. J Arthroplasty 2015;30(04):592-594

43 Jonas SC, Shah R, Mitra A, Deo SD. 5-Year cost/benefit analysis of revision of failed unicompartmental knee replacements (UKRs); not "just" a primary total knee replacement (TKR). Knee 2014; 21(04):840-842

44 Cankaya D, Della Valle CJ. Blood loss and transfusion rates in the revision of unicompartmental knee arthroplasty to total knee arthroplasty are similar to those of primary total knee arthroplasty but are lower compared with the revision total knee arthroplasty. J Arthroplasty 2016;31(01):339-341 